\title{
Éduquer les enfants, discipliner les parents : les rapports famille-école à Montréal, 1910-1960
}

\section{Denyse Baillargeon}

\begin{abstract}
RÉSUMÉ
Se fondant sur des sources émanant du milieu scolaire franco-catholique montréalais, cet article explore la question des rapports entre la famille et l'école. Il entend préciser quelles étaient les attentes des parents vis-à-vis l'école, mais surtout quelles étaient les attentes de l'école vis-à-vis des parents à partir de deux études de cas : la création d'écoles maternelles et la question des devoirs. L'analyse des enjeux que ces deux questions soulèvent, soit la prise en charge des tout petits par le système scolaire et la contribution des parents à la formation scolaire des enfants plus âgés, amène à conclure que le projet éducatif de l'école reposait en grande partie sur la collaboration des parents. Discipliner les parents pour qu'ils se plient aux attentes de l'école a donc fait partie intégrante du projet de scolarisation des enfants, ce à quoi les parents ont souvent cherché à résister.
\end{abstract}

\section{ABSTRACT}

Based on sources drawn from the French Catholic school environment in Montreal, this article explores the question of the relationship between the family and the school. The author intends to clarify what parents expected from the school and, in more detail, what the school expected of parents, based on two case studies: the creation of nursery schools and the question of homework. An analysis of the issues raised by these two questions, namely the school system taking over the care of very young children and the contribution of the parents to the education of older students, leads to the conclusion that the educational project of the school rested largely on the collaboration of the parents. Disciplining the parents so that they comply with the expectations of the school was thus an integral part of schooling the children, something which parents often tried to resist.

\section{Introduction}

Depuis maintenant plusieurs décennies, l'histoire de l'éducation s'est intéressée aux multiples facettes de la formation scolaire et aux visées de ses promoteurs. Sous le regard de cette historiographie, l'école est apparue comme un lieu de reproduction des inégalités sociales et genrées, et aussi comme l'endroit par excellence où les enfants des classes populaires ont été modelés ou remodelés en fonction d'un ensemble de valeurs associées aux classes moyennes. ${ }^{1}$ Loin de se limiter à transmettre des 
connaissances, l'école aurait favorisé l'acquisition de comportements et d'habitudes de vie jugées socialement acceptables ${ }^{2}$. Certains auteurs ont aussi fait remarquer que les instituteurs, et en particulier les institutrices, ont été, tout autant que leurs élèves, la cible de prescriptions venant des autorités scolaires et visant à les constituer en modèle de respectabilité. ${ }^{3}$ Inspirés du concept de régulation morale proposé par Michel Foucault, plusieurs de ces travaux insistent sur les techniques de surveillance et les pratiques disciplinaires mises en œuvre par l'école et sur la "normalisation " de certaines manières d'être et de faire. ${ }^{4}$

S’ils ont parfois été implicitement inclus dans l'équation, les parents, quant à eux, ont plus souvent été laissés dans l'ombre. C'est particulièrement le cas au Québec où mis à part le dossier de la fréquentation scolaire obligatoire, bien peu d'historiens se sont penchés sur la dynamique famille-école. ${ }^{5}$ Les parents constituaient pourtant la première cible des maîtres et des autorités scolaires, car comme se plaisait à le rappeler le clergé catholique québécois, ils étaient les premiers responsables de l'éducation des enfants et ils devaient donc être persuadés de la nécessité de collaborer avec l'école. En fait, plus qu'une simple collaboration, l'école demandait aux parents qu'ils endossent ses visées éducatives et qu'ils se plient aux exigences que cela pouvait comporter. À côté des élèves et des enseignants, les parents apparaissent donc comme un troisième groupe qui a été confronté aux attentes et à la discipline scolaires. Fondé sur le dépouillement de sources provenant du milieu scolaire franco-catholique montréalais, ${ }^{6}$ cet article examine deux études de cas qui en font la démonstration : les écoles maternelles et les devoirs à la maison. L'analyse des enjeux que ces deux questions soulèvent, soit la prise en charge des tout petits par le système scolaire et la contribution des parents à la formation scolaire des enfants plus âgés, montre bien que l'école cherchait à étendre ses procédés de régulation morale jusqu'au sein de la famille, car elle comptait sur un milieu familial réformé pour mener à bien son projet éducatif. Dans le cas des maternelles, il s'agissait pour l'école de se substituer à un milieu familial jugé déficient tout en proposant un modèle alternatif d'éducation qui, espérait-on, ferait figure d'exemple auprès des parents; dans le cas des devoirs, il s'agissait plutôt d'amener les parents à adhérer à une pratique qui exigeait qu'ils encadrent leurs enfants en accord avec les objectifs scolaires.

\section{Les écoles maternelles}

L'histoire de l'enseignement préscolaire n'a pas encore fait l'objet d'étude approfondie au Québec.7 À l'exception de l'article de Micheline Dumont portant sur les salles d'asile des Sœurs grises qui ont fonctionné entre les années 1860 et les années 1920, les historiens québécois de l'éducation ne semblent pas avoir été attirés par le sujet, ni d'ailleurs les historiens canadiens-anglais. ${ }^{8}$ Les Kindergartens, jardins d'enfants, écoles enfantines et autres institutions du même genre ont cependant donné lieu à plusieurs travaux tant en France, aux États-Unis, qu'en Angleterre et en Allemagne. ${ }^{9}$ Il en ressort que, dès la fin du XVIII siècle, plusieurs pays d'Europe ont mis sur pied des institutions visant l'éducation physique, morale et intellectuelle des jeunes enfants, y compris l'apprentissage précoce de l'écriture, de la lecture et du calcul. 
Même si son institution n'est pas la première à se préoccuper de leur scolarisation, Friedrich Fröbel est néanmoins considéré comme le concepteur des véritables écoles maternelles. Contrairement aux autres établissements qui accueillaient des petits, les kindergartens de Fröbel innovent en effet en ce qu'elles proposaient un modèle pédagogique davantage centré sur le jeu et qu'elles visaient surtout à " cultiver " la nature de l'enfant, à l'amener à développer ses capacités et l'expression de son individualité, tout comme la coopération avec les autres. ${ }^{10}$ L'expérience française, mais aussi montréalaise, montre cependant que l'arrivée des « écoles maternelles n'a pas nécessairement mis fin aux tentatives de scolarisation des tout jeunes, même si cette ambition a été de plus en plus vivement condamnée par les éducateurs de la seconde moitié du XIX ${ }^{\mathrm{e}}$ siècle $»{ }^{11}$ D'ailleurs, pour plusieurs auteurs, ce qui différencie le plus distinctement les premières institutions pour enfants des écoles maternelles, c'est moins les activités qu'elles proposent que la nature charitable des premières et le caractère public des secondes.

Les historiens des écoles maternelles, comme des autres institutions infantiles, s'entendent en général pour souligner le but réformateur de ces fondations. Selon les pays et les époques, elles ont parfois eu pour objectif avoué de faciliter l'intégration des mères au marché du travail, mais dans la plupart des cas, elles avaient surtout pour finalité de prendre en charge les enfants des classes populaires et, aux États-Unis surtout, des immigrants afin de les transformer en futurs citoyens responsables. Aux yeux de ses promoteurs, l'école maternelle apparaissait comme " un antidote à la paresse, au mécontentement, au conflit de classe et à l'égoïsme » et comme un moyen privilégié de combattre la désorganisation familiale, la subversion politique et les habitudes de vie des "cultures étrangères ". ${ }^{12}$ À Montréal, comme on l'a déjà souligné, les Sœurs grises avaient déjà ouvert des salles d'asile en s'appuyant sur le modèle de leur contrepartie française. Initiée par le sulpicien Victor Rousselot, lui-même Français d'origine, la première salle d'asile est fondée en 1858; en 1900, on en compte cinq qui reçoivent quelques milliers d'enfants chaque année. ${ }^{13}$ Âgés de trois à sept ans et très majoritairement issus de la classe ouvrière, ils appartiennent à des familles dont la mère est temporairement au travail ou qui habitent des logements trop exigus pour qu'ils puissent y jouer. Sortir les enfants de la rue est d'ailleurs l'une des raisons souvent invoquées pour justifier l'existence des salles d'asile. Leur programme qui visait à « préserver l'innocence des petits enfants, développer leur intelligence, former leur coeur à la vertu et favoriser le développement de leurs forces physiques ${ }^{14}$ prétendait "profiter de ces premières années, encore vierges de mauvaises impressions pour développer tout ce qui est bien dans les instincts de l'enfant $"{ }^{15}$ En principe, il s'agissait donc de forger leur caractère, de leur apprendre les bonnes manières et les bons comportements, de faire, en somme, leur éducation comme l'affirmait Julie Gaudry, la religieuse responsable des salles. En pratique cependant, malgré une volonté affichée de ne pas faire des salles d'asile "de petites écoles ", leur fonctionnement reposait sur une discipline plutôt stricte qui n'est pas sans rappeler l'école primaire et plusieurs des activités proposées constituaient en réalité des leçons de lecture, d'écriture et de calcul ou des exercices de mémorisation et même des exercices militaires qui les habituaient "à une prompte obéissance " et "à la soumission envers les maîtres ". ${ }^{16}$ Même si à 
partir des années 1890, elles se réclament de la méthode Fröbel, les salles d'asile de Montréal, comme les salles d'asile françaises d'ailleurs, conservent donc un caractère plus scolaire que ludique. ${ }^{17}$

C'est au moment même où les salles d'asile commencent à fermer leurs portes, soit au cours des premières décennies du $\mathrm{XX}^{\mathrm{e}}$ siècle ${ }^{18}$, que les autorités scolaires de Montréal débattent de l'opportunité d'ouvrir des écoles maternelles sur leur territoire. Dès 1910, à l'instigation du commissaire Eugène Lafontaine, la Commission des écoles catholiques de Montréal (CÉCM), adopte une résolution demandant au Conseil de l'Instruction publique (CIP) le pouvoir d'établir de telles écoles, permission qu'elle obtient l'année suivante, avant d'entreprendre la construction de deux édifices neufs pouvant les abriter. ${ }^{19}$ L'une d'elles, l'École maternelle Sainte-Anne, est érigée dans le quartier Saint-Charles pour les catholiques anglophones à la demande du curé de la paroisse, le curé Daly, et de l'échevin O'Connell; l'autre est bâtie à l'initiative des commissaires dans le quartier Saint-Eusèbe habité par des franco-catholiques et sera baptisée l'École maternelle Gédéon-Ouimet, du nom du premier ministre et troisième surintendant de l'Instruction publique. ${ }^{20}$ La direction de ces écoles est confiée respectivement aux Sœurs de la Providence et aux Filles de la Sagesse, deux communautés qui oeuvraient dans le domaine de la santé et de l'assistance, plutôt qu'en éducation, ce qui suggère qu'aux yeux des autorités scolaires, la maternelle comportait toujours une dimension charitable. ${ }^{21}$ Des échanges entre les commissaires montrent cependant que le recours à ces communautés a soulevé certaines objections, car certains d'entre eux auraient préféré qu'on les confie à des enseignantes laïques. ${ }^{22}$ Craignait-on que les sœurs n'aient pas les compétences voulues? Les procès-verbaux, plutôt laconiques, ne permettent pas de le conclure, mais il semble évident que l'École Sainte-Anne qui accueillait des enfants âgés de 3 à 6 ans, correspondait mieux au modèle de l'école maternelle que l'École Gédéon-Ouimet qui admettait les enfants jusqu'à la $4^{\mathrm{e}}$ année du primaire. ${ }^{23}$ Ajoutons qu'au moment précis où la CÉCM s'engageait dans la fondation de maternelles, le surintendant de l'Instruction publique n'avait pas encore développé un programme précis concernant ces écoles. Ce n'est qu'en mars 1915, en réponse à une autre requête de la CÉCM, qu'il promit de se pencher sur la question, alors que les deux écoles fonctionnaient depuis l'automne précédent. ${ }^{24}$

Suivant le règlement finalement adopté par le Comité catholique de l'Instruction publique en mai $1915^{25}$, les écoles maternelles étaient définies comme des " établissements de première éducation ", dont le but était de donner aux enfants de 3 à 6 ans des deux sexes « les soins que réclame leur développement physique, intellectuel et moral, comme ils le recevraient dans leur famille, d'une mère intelligente et tendre $»{ }^{26}$ Tout en spécifiant que ces écoles ne devaient pas être transformées en écoles primaires, le nouveau règlement précisait qu'elles devaient s'attacher à inculquer aux enfants "des habitudes de propreté, d'ordre, de politesse et d'obéissance; d'exciter en eux l'activité spontanée; de leur inspirer l'amour du bien et l'horreur du mal; de les soumettre, en un mot, à toutes les influences bienfaisantes d'une éducation maternelle intelligemment comprise $" .{ }^{27}$ Considérée comme une transition entre la famille et l'école, la maternelle était censée reproduire " la douceur affectueuse et indulgente de la famille ", en même temps qu'elle devait "initi[er] au travail et à 
la régularité de l'école $"{ }^{28}$ En guise de programme, le surintendant préconisait les leçons de choses, les causeries, le chant, le dessin, la lecture, le calcul et les récitations qui devaient alterner avec les jeux et les exercices corporels empruntés à la méthode Fröbel. L'enseignement de la lecture, de l'écriture et du calcul, inclus dans le programme pour, disait-on, satisfaire les demandes des parents et surtout recommandé pour les 5-6 ans, devait se résumer à une demi-heure d'exercices matin et soir. ${ }^{29} \mathrm{La}$ formation proprement scolaire devait donc occuper la part congrue du temps passé à la maternelle, mais dans l'esprit du Surintendant, tout en n'étant pas une école comme les autres, la maternelle devait " préparer l'enfant à recevoir avec fruit l'instruction primaire $"{ }^{30}$

Plusieurs éducateurs du début du XXe siècle se plaignaient en effet que les enfants des classes populaires arrivaient à l'école primaire sans préparation aucune, sans que leurs sens et leur intelligence n'aient été éveillés, sans posséder un vocabulaire suffisant ou les connaissances de base essentielles à la bonne compréhension des leçons et des directives du maître, sans même avoir jamais tenu un livre dans leurs mains, et sans qu'ils sachent au moins signer leur nom ou faire le signe de la croix. ${ }^{31} \mathrm{Ce}$ manque de préparation, dont la faute était attribuée tantôt à la négligence et à l'incurie parentales, tantôt au nombre élevé d'enfants par famille, était considéré comme un réel obstacle à une scolarisation réussie des masses populaires. En se fondant sur le jeu pour développer le sens de l'observation, l'imagination et même les connaissances générales, la maternelle était censée " canaliser toutes les énergies naissantes de l'âme enfantine en vue de son éducation ${ }^{32}$ et ainsi favoriser son intégration et sa réussite scolaires. Mais derrière ces objectifs purement éducatifs, se profilait également la volonté d'encadrer le plus tôt possible les enfants de la classe ouvrière. Selon le curé Daly, ces écoles remplissaient en effet un rôle social encore plus important, car elles retiraient "l'enfant du peuple " de la rue et des taudis infects où il était exposé au spectacle « troublant et repoussant (...) des passions populaires et de l'alcoolisme » et au langage grossier et vulgaire de ses aînés, " source de la dégénérescence morale dans nos grandes villes » déplorait-il. ${ }^{33}$ L'école maternelle offrait bien sûr un environnement qui se trouvait aux antipodes de ce milieu délétère :

Ce n'est plus une atmosphère saturée de miasmes et chargée d'influences démoralisantes que l'enfant y respire. Ici tout est hygiénique, moralisateur, éducateur. Instinctivement, l'enfant y apprend l'ordre, la propreté, l'obéissance, la politesse, la piété. Ces idées d'ordre et de respect, ces sentiments élevés, ces aspirations pour une vie plus noble pénètrent dans l'âme enfantine et par elle s'introduisent insensiblement dans le foyer. La mère elle-même subit l'influence éducatrice de l'école maternelle et comprend mieux à son contact son rôle sublime. Aussi, le «Kindergarten» [...] sera un centre de regénération (sic) sociale, parce qu'il s'applique surtout à guérir le mal dans sa racine et à protéger la richesse de la nation dans sa source. ${ }^{34}$

La protection morale des jeunes enfants se retrouvait donc au cœur du projet de création des premières maternelles de Montréal. Bien plus, ses propagandistes caressaient 
l'espoir que les valeurs dont ils feraient l'apprentissage imprègneraient bientôt l'ensemble de la famille et en particulier les mères qui en tireraient des leçons sur la manière d'éduquer leur progéniture. Il ne s'agissait donc pas seulement de former ou de réformer les enfants dès leur plus jeune âge, mais aussi de discipliner les parents en diffusant jusque dans la famille les idéaux proposés par l'école. Même si les autorités religieuses et scolaires n'avaient de cesse de répéter que les parents étaient les premiers responsables des enfants et que l'école maternelle ne devait pas viser à les remplacer, mais uniquement à les aider ${ }^{35}$, dans les faits, les parents des classes populaires étaient généralement considérés comme des incapables qui compromettaient l'éducation de leurs enfants par leur ignorance, leur incurie ou leur parfaite insouciance. Davantage qu'une aide à la famille, la maternelle était elle-même présentée comme une famille de substitution, une "famille agrandie ${ }^{36}$ plus à même de développer les facultés de l'enfant que sa famille d'origine. C'est d'ailleurs pour mieux reproduire le milieu familial que les maternelles accueillaient des enfants des deux sexes, ce qui n'était pas le cas de l'école primaire. L'organisation de la classe, l'ameublement et le décor devaient pour leur part ressembler à " un foyer, un 'home' idéal » disait le curé Daly où rayonnerait la « douce atmosphère du chez soi ». Quant aux institutrices, elles étaient constamment comparées à des mères, ou plutôt à la mère idéalisée que les éducateurs auraient aimé voir à la tête de chaque foyer, une mère intelligente, aimante, patiente, attentive aux besoins de son enfant et capable de le guider dans son développement cognitif et affectif. ${ }^{37}$

Comme le soulignait le commissaire Lafontaine, la fondation de deux écoles maternelles par la CÉCM en 1914 s'inscrit dans un contexte où la société québécoise se préoccupait de plus en plus du sort de ses enfants et où elle avait mis en place de multiples institutions - gouttes de lait, hôpitaux pédiatriques, crèches, parcs et terrains de jeux, colonies de vacances, tribunaux pour jeunes délinquants, etc. — qui cherchaient à " sauver l'enfant en l'arrachant à tous les dangers qui mena[çaie]nt sa frêle existence " et à lui donner " toutes les facilités pour atteindre le haut degré de développement physique et intellectuel et moral $" .^{38}$ Les tentatives pour développer un véritable réseau d'écoles maternelles, comme l'aurait souhaité Lafontaine, ${ }^{39} \mathrm{sem}$ blent cependant avoir tourné court : apparemment débordée de demandes pour la construction de nouvelles écoles primaires, la CÉCM n'a pas été en mesure de multiplier leur nombre. ${ }^{40}$

La courte vie des premières écoles maternelles montréalaises n’a pas mis fin au débat sur la nécessité de ces écoles, mais à compter des années 1930, la question commence à se poser différemment. D'une institution visant à réformer les enfants des quartiers ouvriers, et éventuellement leur famille, la maternelle apparaît alors comme une étape fondamentale dans le processus de scolarisation normal de tous les enfants. Claudine Vallerand, fondatrice de la première maternelle privée de Montréal (1938) et de l'École des parents du Québec (1939), ${ }^{41}$ devient alors l'une des principales propagandistes de l'école maternelle pour tous. Dès 1939, dans un article publié dans La Revue Dominicaine, Vallerand souligne les avantages qu'en retirent les enfants du point de vue de leur développement psychologique, moteur et intellectuel. À son avis, l'école maternelle constitue la " première formation de la personnalité » qui renforce 
" la possibilité physique de grandir, la puissance d'apprendre, la force morale de dominer. [...] c'est le milieu où l'enfant acquerra [...] l'habitude d'écouter, de voir, d'imiter, d'observer, [...] les habitudes de propreté, d'ordre, de méthode dans le travail, de politesse, d'attention, d'activité intellectuelle [...] C'est la base indispensable sur laquelle s'érigera tout le reste de l'éducation $» .^{42}$

À première vue, on peut donc dire que Vallerand prêtait à l'école maternelle les mêmes avantages que ses premiers promoteurs à ceci près cependant que pour elle la maternelle demeurait un lieu d'éducation et non pas d'instruction et que sa philosophie s'appuyait très explicitement sur les théories psychologiques qu'elle a largement contribué à répandre dans le Québec d'après-guerre. ${ }^{43}$ Selon elle, la vraie maternelle se distinguait de la fausse en ce qu'elle récusait les apprentissages formels qui maintenaient les enfants dans une attitude passive et qu'elle laissait l'enfant évoluer « en toute liberté, sans autre contrainte que la discipline qu'il s'impose, qu'il accepte spontanément $" .{ }^{44}$ En fait, la maternelle devait répondre à tous les besoins de l'enfant afin de favoriser l'acquisition d'une personnalité stable, ce qui en retour assurerait la « formation d'une nation saine et bien équilibrée ". ${ }^{45}$ La complexité de cette tâche était telle qu' elle ne pouvait plus être confiée uniquement aux mères de familles, car même le « milieu familial le plus sain, le mieux ordonné " ne pouvait prétendre combler tous les besoins de l'enfant. ${ }^{46}$ Pour Vallerand, éduquer les enfants exigeait désormais une expertise psychologique que les mères de famille ne détenaient pas nécessairement; la maternelle dirigée par des éducatrices bien formées et compétentes s'avéraient donc un " complément indispensable à l'éducation de famille $» .{ }^{47}$ Compte tenu de ses objectifs nationaux, elle aurait dû accueillir les enfants " de toutes conditions ". ${ }^{48}$ Dans les nombreux textes qu'elle publiera par la suite, notamment dans La Revue de l'École des Parents organe de l'association du même nom publiée à partir de 1949, Vallerand, de même que quelques jardinières, continueront de prôner la maternelle pour tous, tout en insistant sur la véritable nature et les finalités réelles de la formation préscolaire des petits, à savoir assurer le développement psycho-moteur et intellectuel des enfants par le jeu. ${ }^{49}$

Des années 1930 aux années 1960, alors que la CÉCM commence à mettre en place ce type d'écoles, la fréquentation de l'école maternelle a donc été défendue non pas par les autorités scolaires, mais par une représentante de la classe moyenne canadienne-française, elle-même propriétaire d'une maternelle privée et fondatrice d'une organisation de parents qui cherchait à se positionner comme partenaire de l'école. Outre la diffusion des théories psychologiques, l'École des Parents (ÉDP) s'était en effet donné pour mandat de "sauvegarder les droits de la famille sur l'enfant " ${ }^{50}$ en exigeant que l'école réponde aux attentes parentales — du moins telles que l'ÉDP les concevait. Parmi les réformes souhaitées figurait un enseignement mieux adapté aux besoins d'une société moderne tant en ce qui concerne les matières au programme que les méthodes pédagogiques ${ }^{51}$ et fondé sur les connaissances apportées par la psychologie au sujet du développement émotif et cognitif des enfants. L'instauration de maternelles constituait le socle sur lequel devait reposer ce nouvel édifice éducatif, mais plus largement, le programme de l'ÉDP supposait que la famille contribue de manière active à la formation des enfants, futurs citoyens, dès leur plus jeune âge 
et qu'elle-même se réforme pour répondre à ces nouvelles exigences. "S'il doit y avoir des réformes en éducation ", affirmait Claudine Vallerand, " elles doivent naître d'abord [...] au sein même de la famille " par une prise de conscience des parents de leur "responsabilité envers [les] enfants et envers [la] nation " et par une réforme de leurs " méthodes d'éducation ". ${ }^{52}$ Tandis que les premières maternelles de la CÉCM visaient les classes défavorisées, celles que Vallerand aurait aimé voir s'implanter ciblaient l'ensemble des enfants, car selon le discours psychologique, chacun d'eux nécessitait un environnement idéal pour se développer pleinement. Il ne s'agissait donc plus de contrer la dégénérescence sociale en inculquant les valeurs des classes dominantes aux enfants pauvres, mais de façonner une nation de citoyens équilibrés en suivant les préceptes des psychologues, ce qui engageait l'ensemble des mères et des familles. Plus encore, si au début du siècle, la maternelle était considérée comme un instrument d'éducation des mères des familles, dans l'après-guerre, Vallerand estimait qu'à défaut de maternelles, les mères devaient prendre la relève de l'école en organisant une maternelle chez elles. ${ }^{53}$ Compenser un système scolaire jugé déficient lui apparaissait comme un des devoirs de la famille. Dès années 1930 aux années 1960, on peut donc dire que c'est un groupe de parents regroupés dans l'ÉDP qui ont, par l'entremise de Claudine Vallerand, prôné la scolarisation précoce des enfants, l'éducation préscolaire leur apparaissant comme un signe de modernité à laquelle ils voulaient convertir l'école et l'ensemble des familles pour édifier une société pénétrée des valeurs associées à la démocratie libérale.

\section{Les devoirs scolaires}

Les devoirs scolaires représentent un autre dossier qui permet d'explorer la dynamique famille-école. Depuis les années 1920 jusqu’à la fin des années 1950, la question a en effet suscité de nombreuses polémiques qui permettent d'examiner les attentes de l'école vis-à-vis des parents et réciproquement. Les devoirs devaient-ils être faits à domicile ou à l'école ou devait-on même tout simplement les abolir? Voilà pour l'essentiel les termes dans lesquels se posait le débat. Comme on le verra, les arguments avancés en faveur des devoirs à domicile n'ont guère varié au fil des décennies; dans l'ensemble ils montrent bien que, derrière des objectifs pédagogiques, se cachait une volonté de discipliner à la fois les enfants et les parents et que ces derniers avaient leurs propres raisons pour se montrer, ou non, favorables aux devoirs.

Une pétition, datant de 1914 et signée par une quarantaine de parents d'élèves de la $7^{\mathrm{e}}$ et de la $8^{\mathrm{e}}$ années de l'École Frontenac pour demander que leurs enfants « aient, [...], le privilège de faire leurs devoirs écrits dans leur classe respective " ${ }^{54}$, montre que les devoirs ont très tôt fait l'objet de revendications parentales. C'est cependant dans les décennies suivantes que le débat autour de cette question s'est véritablement enclenché. En 1924, en réponse à la montée d'une certaine opposition de la part des parents, la revue du Département de l'Instruction publique (DIP), L'enseignement primaire, faisait paraître un article exposant les buts poursuivis par les devoirs à domicile, de manière à mieux justifier leur existence, tandis que l'année suivante, l'abbé J. O. Maurice consacrait l'une de ses causeries pédagogiques à ce sujet. Dans les 
deux cas, les auteurs soutenaient que les devoirs à domicile servaient à consolider les apprentissages faits en classe, mais qu'ils représentaient aussi un excellent moyen d'inculquer chez l'enfant l'habitude du travail, de cultiver son sens des responsabilités et son autonomie. ${ }^{55}$ À ceux qui estimaient que les devoirs constituaient une charge trop lourde pour la santé physique et mentale des élèves déjà épuisés par la journée passée en classe, l'abbé Maurice répondait que le jeu fatiguait tout autant les enfants et que la fatigue n'avait rien d'anormal en soi « elle est même salutaire » ajoutait-il « et l'école ne doit pas craindre de l'imposer et d'y habituer les enfants ". ${ }^{56}$ Plus encore, à son avis, et on touche là un des arguments de fond des tenants des devoirs à domicile, il n'était pas bon que les enfants aient trop de loisirs, car à mesure qu'ils grandissaient, ils cherchaient des distractions en dehors de la maison où ils s'exposaient " presque infailliblement à toutes sortes de contacts ou de spectacles dangereux. Ne vaut-il pas mieux qu'une besogne scolaire modérée, utile et qu'on sait rendre attrayante, vienne les retenir au logis? ", demandait-il. ${ }^{57}$

Ainsi, dans l'esprit de ce pédagogue, les devoirs à domicile servaient des fins éducatives non seulement en raison de la formation qu'ils permettaient à l'élève d'acquérir, mais aussi, et peut-être surtout, en vertu des dangers moraux qu'ils lui évitaient. Maurice estimait par ailleurs que l'opposition des parents aux devoirs à domicile, qu'elle ait été inspirée par la tendresse envers leurs enfants ou parce qu'ils avaient besoin d'eux pour rendre de menus services, devait tout simplement être balayée car il revenait à l'école de statuer sur les questions relevant de la pédagogie. De son point de vue, les devoirs à domicile représentaient une belle occasion d'amener les parents à s'intéresser aux études de leurs enfants et de collaborer avec les maîtres et il allait jusqu'à dire que même si les devoirs à domicile ne permettaient d'atteindre que ce dernier but, ils vaudraient la peine d'être maintenus. ${ }^{58}$

Quelques années plus tard, réagissant aux propos du Directeur de l'enseignement protestant de la province qui prônait l'abolition des devoirs à domicile, Emma-Maria Lalonde, enseignante à l'École normale de Valleyfield, prenait elle aussi la plume pour se porter à la défense d'une tradition que d'aucuns, disait-elle, voulaient abandonner au nom d'un " amour exagéré du bien-être ". ${ }^{59}$ S'inspirant d'un article publié dans L'Enseignement primaire en 1884, Lalonde tentait de démolir les arguments des «partisans de l'école agréable » en réitérant que les devoirs à la maison renforçaient les apprentissages, forçaient l'enfant à demeurer attentif en classe et, surtout, les initiaient " à la grande loi du travail ». En abandonnant leurs jeux pour faire leurs devoirs les enfants s'habituaient " de bonne heure à faire alterner l'accomplissement du devoir avec les charmes de la distraction, à concilier les exigences de la vie avec une tendance instinctive au plaisir "; une fois devenus adultes, ils sauraient " faire une juste part à l'agréable et ne considérer en toutes choses que leur véritable perfectionnement ". ${ }^{60}$ Fidèle à la pensée de ses maîtres du XIX ${ }^{\mathrm{e}}$ siècle, elle considérait que les devoirs contribuaient grandement à « l'éducation morale » des enfants, car ils permettaient de développer les qualités nécessaires au bonheur des humbles : " ordre, régularité, propreté, économie, courage, persévérance, soumission ${ }^{6}{ }^{61}$

Tout comme pour la maternelle, la pratique des devoirs à la maison visait donc bien d'autres buts que la seule formation scolaire des enfants. Leur édification, leur 
encadrement moral, et plus particulièrement l'acquisition de valeurs et de comportements susceptibles d'en faire des citoyens et des travailleurs fiables, responsables et respectueux de l'autorité, représentaient des objectifs tout aussi fondamentaux que le renforcement des connaissances transmises en classe. Plus encore, les devoirs scolaires devaient également servir à inciter les parents à s'intéresser à la scolarisation de leurs enfants. Souvent dénoncée, la tendance des parents à s'en remettre entièrement à l'école était loin de plaire aux éducateurs, car pour accomplir leur mission ils avaient besoin de la collaboration parentale. En effet, c'est uniquement dans la mesure où les parents appuyaient les enseignements et les préceptes moraux des maîtres que l'école pouvait espérer modeler les enfants suivant ses aspirations. Dans cette perspective, l'intérêt que les parents accordaient aux devoirs des enfants, l'insistance qu'ils mettaient à ce que les devoirs soient bien faits, la surveillance qu' ils exerçaient auprès des enfants faisant leurs devoirs devenaient autant de marques d'appui envers les maittres, ce qui renforçait l'autorité de ces derniers auprès des élèves. ${ }^{62}$ Discipliner les parents pour qu'ils disciplinent les enfants afin de les préparer à se soumettre à l'autorité de l'école et à ses enseignements, voilà l'objectif beaucoup plus ambitieux auquel contribuaient les devoirs scolaires faits à la maison.

Comme le déploraient les partisans de la collaboration famille-école, tous les parents n'étaient pas enthousiastes à l'idée de superviser les devoirs de leurs enfants et plusieurs ne se gênaient pas pour dénoncer la charge excessive de travail que les maîtres imposaient aux écoliers. Ainsi, dans une lettre envoyée au directeur des études de la CÉCM en 1930, un père de famille affirmait que les devoirs étaient " une horreur ", une " abominable plaie " dont il le priait de bien vouloir le délivrer. ${ }^{63}$ Quatre ans plus tard, une autre correspondante condamnait la longueur des devoirs imposés à sa fille et prédisait que si la CÉCM ne donnait pas le mot d'ordre de mettre fin à ce genre d'abus, bien des parents anglo-catholiques, communauté à laquelle elle appartenait, finiraient par envoyer leurs enfants à l'école protestante, moins exigeante sur ce point. ${ }^{64}$ L'année suivante, un couple se plaignait de la longueur des devoirs et du manque de clarté des directives données par les maîtres : " qui est-ce qui en souffre ce sont nous les parents qui le soir venu sommes hantés de questions sur des choses que nous avons appris il y a vingt et trente ans et qui sont par conséquent oubliées ". Disant espérer que leur lettre "serve pour tous les parents de notre avis ", ils demandaient qu'une heure d'étude soit ajoutée à la fin de la classe régulière "afin qu'à la maison, nous les parents puissions avoir la paix et la tranquillité $»{ }^{65}$

La crise économique qui a sévi tout au long des années 1930 a eu pour effet de nourrir l'opposition aux devoirs à domicile. Dans un contexte où les difficultés financières obligeaient les familles à s'entasser dans des logements trop étroits, ou pire encore à subir des coupures de courant, l'obligation pour les enfants de faire des devoirs à la maison apparaissait comme un fardeau insurmontable et même comme une véritable injustice aux yeux de parents, de représentants syndicaux et même d'un échevin. En 1935, ils ont réclamé et obtenu l'abolition des devoirs et des leçons pour les plus jeunes et l'ajout d'une période d'étude pour les plus âgés, du moins pour un temps. ${ }^{66}$ Deux ans plus tard, en effet, la CÉCM décidait de reprendre la pratique des devoirs à domicile sous prétexte que le temps alloué aux devoirs en classe n'était pas 
suffisant pour que les élèves puissent compléter tous leurs travaux scolaires et que ceux qui avaient le temps de finir leurs exercices écrits refusaient d'apporter leurs manuels à la maison, négligeant ainsi leurs leçons. Mais les commissaires semblaient surtout s'inquiéter du temps libre que la pratique des devoirs à l'école laissait aux enfants : «Les élèves sont dans la rue plus que jamais, il n’y a pas à le nier. Cette oisiveté depuis quatre heures et quart de l'après-midi est plus dommageable que les quelques inconvénients que peuvent présenter les devoirs à la maison $"{ }^{67}$ affirmaient-ils.

C'est donc pour des raisons d'ordre pédagogique, mais aussi pour éviter le " flânage » et les risques de délinquance que cela pouvait entraîner que les commissaires ont remis à l'honneur les devoirs à la maison. Pourtant, selon une étude réalisée au nom de la commission scolaire au moment de réintroduire les devoirs à la maison, bien des parents préféraient la pratique des devoirs à l'école en raison de conditions matérielles défavorables, de l'atmosphère bruyante du foyer, mais aussi parce qu'ils estimaient que la surveillance des devoirs faisait partie intégrante de l'instruction de leurs enfants, une responsabilité qui revenait à l'école et non pas aux familles. Pour ces parents, il était déjà bien suffisant de se charger de l'éducation, un point de vue que l'école, qui faisait souvent grand cas de leurs droits, aurait dû respecter. ${ }^{68}$ Des lettres de protestations provenant notamment du Congrès des métiers et du travail de Montréal et du Cercle ouvrier Delorimier, qui alléguant, entre autres, le manque d'instruction des parents et leur incapacité à aider leurs enfants, réclamaient également le maintien de la période d'étude, mais ce fut peine perdue. ${ }^{69}$ À partir de 1945 , l'expérience de l'étude à l'école après la classe a été reprise pour certains groupes d'élèves avant d'être abandonnée de nouveau en $1949,{ }^{70}$ en même temps que la CÉCM décidait "d'entreprendre une campagne intense de publicité, par tous les moyens jugés opportuns, dans le but de rappeler aux parents l'intérêt qu'ils doivent porter aux études de leurs enfants et d'assurer dans le domaine de l'instruction et de l'éducation, la parfaite collaboration entre la famille et l'école ${ }^{71}{ }^{71}$

Pendant que la CÉCM abolissait la période d'étude après la classe, les autorités scolaires à l'extérieur de Montréal ont commencé à s'inquiéter des conditions dans lesquelles les enfants devaient faire leurs devoirs à la maison et à s'interroger sur leur pertinence. ${ }^{72}$ Ainsi, une enquête menée dans la région de Mont-Laurier révélait que $65 \%$ des enfants sondés faisaient leurs devoirs sur la table de la cuisine et que $58 \%$ se disaient incommodés par la radio, les pleurs et les cris des enfants plus jeunes ou les conversations de leurs parents et de leurs aînés; bien plus, 48 \% avouaient faire un autre travail en même temps que leurs devoirs, le plus souvent assurer la surveillance de leurs frères et sœurs plus jeunes, si bien que $54 \%$ disaient qu'ils auraient préféré faire leurs devoirs à l'école de manière à avoir un peu de tranquillité. ${ }^{73}$ Commentant ce sondage, Rolande Major Charbonneau, une pédagogue membre de l'ÉDP, se demandait si « le foyer [était] bien un endroit propice à la réflexion, à l'étude, en un mot au travail intellectuel ${ }^{74}$ En 1950 , le Comité catholique de l'Instruction publique demandait l'opinion de trois éducateurs qui, au final, se montrèrent plutôt favorables au maintien des devoirs à la maison, quitte à en modifier la teneur et la longueur. Selon Michel Savard, inspecteur général des écoles primaires de la province, les conditions matérielles de la plupart des familles n'étaient pas si déplorables qu'elles 
empêchaient tout devoir à domicile et de toute façon, ceux-ci étaient essentiels, car ils représentaient un des moyens privilégiés d'intéresser les parents aux études de leurs enfants : " nous aurons vite fait d'éteindre l'intérêt des parents pour l'école, lorsque nous aurons supprimé entièrement les devoirs à domicile ", affirmait-il. ${ }^{75}$ Faisant état d'une autre enquête réalisée à Rimouski au début de l'année 1950, Paul Hubert, inspecteur régional pour le DIP, concluait pour sa part que les instituteurs, les inspecteurs d'écoles et $95 \%$ des parents de cette région rurale s'entendaient pour réclamer des devoirs à domicile, mais suivant une formule allégée et améliorée. Les instituteurs y tenaient pour établir " une correspondance entre la famille et l'école ", tandis que les parents jugeaient qu'ils étaient indispensables pour que leurs enfants apprennent mieux, mais aussi, parce qu'ils permettaient de surveiller les maîtres : «Par les devoirs, je vois si nous avons une maîtresse qui enseigne bien " disait par exemple l'une des mères sondées. Autre utilité des devoirs selon certains parents : "ils empêchent [les enfants] de courir le soir $"{ }^{76}$ Ainsi, tout en étant un moyen de contrôle de l'école sur la famille, les devoirs pouvaient s'avérer un moyen pour la famille de contrôler l'école et un prétexte commode pour retenir les enfants à la maison, preuve que certains parents partageaient l'avis des éducateurs au sujet des effets néfastes de l'oisiveté. Mais plusieurs admettaient que leur absence facilitait grandement la tâche des mères de familles qui n'avaient plus à chicaner et à sévir pour que leurs enfants ouvrent finalement leurs livres et leurs cahiers. Surtout, tous les parents insistaient pour que les devoirs soient courts et bien expliqués afin que l'enfant sache très précisément quoi faire. ${ }^{77}$ En d'autres termes, tout en étant d'accord pour superviser les devoirs à la maison, les parents ne voulaient pas que l'école compte sur eux pour compléter l'enseignement reçu en classe ou que ces exercices empiètent de manière exagérée sur la vie familiale ou empêchent les enfants de contribuer aux tâches domestiques.

Cette bonne volonté parentale n'était d'ailleurs pas universelle; une enquête réalisée auprès de 5600 foyers québécois par la Ligue ouvrière catholique (LOC) en 1951, soit l'étude la plus ambitieuse et celle qui s'est le plus intéressée aux familles ouvrières, constatait pour sa part que bien peu de parents se souciaient de savoir si les enfants avaient fait leurs devoirs ou étudié leurs leçons. En fait, dans $15 \%$ des cas, les parents ne savaient même pas si les enfants avaient ou non des devoirs à faire ou des leçons à apprendre. ${ }^{78}$ Selon la LOC, cette indifférence face aux études des enfants se révélait aussi dans le fait que dans plus de $20 \%$ des ménages la radio restait allumée pendant que les enfants travaillaient, une attitude qu'elle qualifiait d'égoïste : "Chacun veut vivre sa petite vie sans songer aux autres, surtout sans songer à l'éducation des enfants. "Ils vont à l'école pour étudier, qu'ils ne nous dérangent pas à la maison!" On oublie souvent que l'école n'est que le complément de la famille et que cette dernière est la première responsable de l'éducation des enfants $" .{ }^{79}$ En conclusion, la LOC disait espérer que « les foyers chrétiens collaborent pour corriger les influences externes qui distraient les pères et mères de famille de leurs devoirs et de leurs responsabilités ». ${ }^{80}$ Bref, les parents devaient apprendre à se discipliner eux-mêmes pour mieux encadrer leurs enfants et ainsi se montrer dignes de leur tâche de premiers éducateurs.

L'ÉDP, qui a aussi mené une enquête auprès des lecteurs de sa revue en 1951, constatait pour sa part que les parents étaient très majoritairement favorables aux 
devoirs (80\%), mais pour de mauvaises raisons ${ }^{81}$ Claudine Vallerand se disait en effet " étonnée et déçue " que bien des parents approuvent les devoirs à domicile parce que " cela les [enfants] tient occupé (sic) » ou parce qu' "il faut qu'ils aient quelque chose à faire ". ${ }^{82}$ À son avis, ces motifs faisaient la preuve d'une totale incompréhension des besoins de l'enfant, au profit d'une volonté " de [le] tenir rivé à sa table de travail ", afin qu'il ne soit pas "une cause de surveillance ". ${ }^{83}$ S'appuyant sur les méthodes promulguées par l'école active, Vallerand allait jusqu'à dire que les « loisirs [étaient] beaucoup plus formateurs [...] que toutes les heures d'étude » et elle enjoignait les parents à « organiser le foyer, la vie familiale et la maison pour que les enfants puissent y trouver l'atmosphère de détente, de recueillement et de joyeux épanouissement ", de sorte qu'il ne soit plus nécessaire de les astreindre à de longues heures d'études sous le fallacieux prétexte de les tranquilliser. ${ }^{84}$ Pour Vallerand, donc, l'absence de devoir ou leur allègement ne signifiaient pas un total désengagement des parents auprès de leurs enfants, bien au contraire. Premiers garants du développement cognitif et de l'équilibre émotif des enfants, les parents devaient assumer pleinement ces responsabilités en devenant les maîtres d'œuvre des loisirs de leurs enfants afin qu'ils en tirent le maximum de bénéfices; dans cette perspective, l'absence ou la diminution des heures consacrées aux devoirs devaient être compensées par un nombre d'heures équivalent de loisirs organisés et supervisés par les parents.

Moussée par les différents sondages réalisés au tournant des années 1950, la question des devoirs à la maison a fait l'objet de nombreux articles dans la revue de l'Alliance des professeurs de Montréal, L'Enseignement, tout au long de l'année 1951. Dans une série de textes intitulée "Les devoirs à domicile : Pour ou contre? ", cette publication faisait état des débats en cours et ouvrait ses pages aux opinions de ses abonnés, essentiellement des enseignants. Malgré les doutes que certains exprimaient au sujet de l'utilité des devoirs à domicile en termes de résultats scolaires, la majorité d'entre eux se disaient favorables aux devoirs à la maison autant pour des raisons pédagogiques que pour cimenter les rapports famille-école. ${ }^{85}$ Certains estimaient toujours que " lors même que ces différents travaux n'auraient d'autres résultats que d'aider les parents à garder leurs enfants au foyer quelques heures de plus chaque jour en leur évitant les vagabondages jusqu'à des heures tardives dans les rues, ruelles, lieux d'amusement publics tels que patinoires, sports divers, théâtres, ainsi qu'un lot d'extravagances chez les plus âgés, il serait avantageux de les maintenir $»{ }^{86}$ Emma-Maria Lalonde, toujours favorable aux devoirs à domicile, soulignait que si ces derniers constituaient une corvée pour les parents, la surveillance " des jeux et des amusements trop prolongés de leurs enfants " représentait une charge encore plus lourde. ${ }^{87}$ En somme, en 1950 comme en 1930, bien des éducateurs étaient prêts à défendre les devoirs comme une panacée à l'oisiveté, la paresse et la délinquance et comme un moyen de forcer les parents à prendre des responsabilités que plusieurs auraient volontiers laissées à l'école. 


\section{Conclusion}

Instruire les enfants n'a jamais été le seul but de l'école publique. Tout en leur enseignant les rudiments de lecture, d'écriture et de calcul, l'école avait (et a toujours) le projet de leur inculquer un ensemble de valeurs pour en faire des citoyens responsables et respectables et des êtres adaptés aux conditions que la société environnante leur réservait. Cette volonté de modeler les jeunes individus supposait que les parents collaborent avec l'école, car pour atteindre pleinement cet objectif, il importait d'exposer les élèves aux mêmes préceptes et aux mêmes exigences à la maison comme dans le milieu scolaire. Discipliner les parents pour qu'ils disciplinent leurs enfants, se substituer à eux au besoin, a donc fait partie intégrante de la mission que l'école s'est assignée. Certes, l'histoire des maternelles montréalaises montre que ce projet a pu être handicapé par des contraintes financières qui ont forcé les autorités scolaires à privilégier d'autres priorités. Il reste que les promoteurs de ce genre d'école y voyaient un moyen de compenser les lacunes d'une éducation familiale jugée déficiente, d'abord au plan moral et plus tard au plan psychologique, et d'amener les parents à faire eux aussi de nouveaux apprentissages. Pour leur part, les débats qui ont périodiquement ressurgi au sujet des devoirs laissent voir qu'ils étaient conçus autant comme un moyen d'approfondir l'enseignement dispensé aux jeunes, que de contrer la délinquance et de maintenir l'intérêt des parents pour les études de leurs enfants. Ces deux exemples rappellent qu'au-delà de l'élève, le véritable interlocuteur de l'école est la famille sur laquelle elle a sans cesse tenté d'exercer une forme de contrôle, ce qui ne veut pas dire qu'elle y soit parvenue facilement ou parfaitement. Dans les milieux populaires notamment, les exigences du quotidien passaient bien avant la surveillance étroite que l'école aurait voulu que les parents exercent sur leurs enfants et sur leurs devoirs. À ce dernier chapitre, des parents et certains groupes sociaux, notamment les syndicats, ont parfois ouvertement contesté les demandes de l'école, tandis que d'autres les endossaient, tout en souhaitant des aménagements afin d'éviter que l'école n'envahisse tout l'espace familial.

Les débats qui ont eu cours sur ces deux questions montrent aussi que des groupes associés au mouvement familial qui s'est développé au Québec à compter des années 1930, comme l'ÉDP et la LOC, ont cherché à convaincre les parents des bienfaits de la maternelle et de la nécessité de superviser les devoirs, ce qui veut dire que l'école pouvait compter sur des alliés provenant de la société que nous qualifierions aujourd'hui de civile. Réunissant des membres de la classe moyenne et de la classe ouvrière respectable, ces associations qui se présentaient comme des porte-parole des parents, prétendaient leur redonner un pouvoir qu'ils avaient perdu aux mains des instances politiques et scolaires, mais elles cherchaient aussi à leur transmettre des savoirs qui leur permettraient d'assumer leur rôle suivant un idéal familial et parental très semblable à celui promulgué par l'école. En d'autres termes, tout en disant vouloir donner une voix aux parents, ces associations adhéraient néanmoins aux visées du système scolaire et ont ainsi contribué à " normaliser » un modèle familial favorable à une étroite collaboration entre la famille et l'école. Par ailleurs, il faut aussi souligner que certains parents voyaient dans les devoirs un moyen privilégié d'évaluer les 
maîtres, ce qui signifie que l'école n'était pas à l'abri d'une forme de contrôle parental. En d'autres termes, si les deux études de cas analysés ici montrent que les parents ont dû s'accommoder de l'école beaucoup plus que cette dernière n'a cherché à répondre à leurs besoins et à leurs aspirations, il reste que les résistances familiales ou la volonté de certains parents de surveiller l'école témoignent des rapports à double sens qui se sont développés entre ces deux institutions fondamentales pour la formation des enfants. Des études complémentaires portant sur des questions, des régions ou des époques différentes permettraient sans doute d'en explorer toute la complexité.

\section{Notes}

1 Une abondante historiographie existe sur ces questions. Voir les bilans de Wendy Johnston, L'école primaire supérieure et le High School public à Montréal, 1920 à 1945, thèse PhD (histoire) Université de Montréal, 1991, 14-30 et Paul Axelrod, "Historical Writing and Canadian Education from the 1970s to the 1990s ", History of Education Quarterly 36, 1 (Spring 1996) : 19-38.

2 Voir entre autres Mona Gleason, "Race, Class, and Health : School Medical Inspection and 'Healthy Children' in British Columbia, 1890-1930 ", Canadian Bulletin of Medical History/Bulletin canadien d'histoire de la médecine 19, 1 (2002) : 95-112; Denyse Baillargeon, " "Une opposition regrettable" : L'inspection médicale des écoliers à Montréal, 1920-1960 ", à paraître dans les actes du colloque Modernité, citoyenneté, déviances et inégalités. Pour une analyse comparative des difficultés du passage à la modernité citoyenne (Cordoue, avril 2006); Martin Pâquet et Jérôme Boivin, « La mesure fait loi. La doctrine de l'hygiène mentale et les tests psychométriques, au Québec pendant l'entre-deux-guerres ", CHR 88, 1 (mars 2007) : 149-179; Alison Prentice, The School promoters. Education and Social Class in mid-nineteenth Century Upper Canada, (Toronto: University of Toronto Press, 2004); Bruce Curtis, Building the Educational State. Canada West, 1836-1871 (London: Althouse Press, 1988) et Andrée Dufour, Tous à l'école. État, communautés rurales et scolarisation au Québec de 1826 à 1859 (LaSalle : Hurtubise HMH, 1996).

3 Susan E. Houston et Alison Prentice, Schooling and Scholars in Nineteenth Century Ontario (Toronto: UTP, 1988); Harry Smaller, "Regulating the Regulators : The Disciplining of Teachers in Nineteenth-Century Ontario", in Kate Rousmaniere, Kari Dehli et Ning de Coninck-Smith, Discipline, Moral Regulation and Schooling. A Social History (New York : Garland Publ., 1997), 87-116. Voir aussi Andrée Dufour et Micheline Dumont, Brève histoire des institutrices au Québec de la Nouvelle-France à nos jours (Montréal : Éditions du Boréal, 2004).

4 Michel Foucault, Surveiller et punir. La naissance de la prison (Paris : Gallimard, 1979).

5 Dominique Marshall, Aux origines sociales de l'État providence (Montréal : PUM, 1997); Thérèse Hamel, «L'obligation scolaire au Québec : enjeu pour le mouvement syndical agricole ", Labour/Le Travail 17 (printemps 1986) : 83-102; Chad Gaffield et Gérard Bouchard, "Literacy, Schooling, and Family Reproduction in Rural Ontario and Quebec " Historical Studies in Education 1 (automne 1989) : 201-218. Sur l'histoire du système scolaire anglo-protestant au Québec et ses liens avec la communauté, voir Roderick MacLoed et Mary Anne Poutanen, A Meeting of the People. School Boards and Protestant Communities in Quebec, 1801-1998 (Montreal : MQUP, 2004).

6 Il s'agit principalement des procès-verbaux des réunions des commissaires de la Commission des Écoles catholiques de Montréal, des dossiers relatifs aux devoirs conservés dans les archives de cette institution, d'une brochure de l'École sociale 
populaire consacrée aux maternelles et des revues L'Enseignement primaire, L'École canadienne, L'Enseignement, L'École des Parents et Le Front ouvrier.

7 Robert Gagnon aborde brièvement cette question dans Histoire de la Commission des écoles catholiques de Montréal. (Montréal : Boréal, 1996), 100-114. Voir aussi Jocelyne Morin, La maternelle. Histoire, fondements, pratiques (Montréal : Gaëtan Morin éditeur/ Chenelière éducation, 2007, $2^{\mathrm{e}}$ éd.).

8 Micheline Dumont, "Des garderies au XIX siècle: les salles d'asile des sœurs Grises à Montréal ", dans Nadia Fahmy-Eid et Micheline Dumont, Maîtresses de maison, maîtresses d'école. Femmes, familles et éducation au Québec (Montréal : Boréal 1983), 261-285.

9 Voir par exemple Ann Taylor Allen, "Let Us Live with our Children : Kindergarten Movements in Germany and the United-Sates, 1840-1914", History of Education Quarterly 28, 1 (spring 1988) : 23-49 et "Gardens of Children, Gardens of God: Kindergartens and Day-Care Centers in Nineteenth-Century Germany", Journal of Social History 19 (spring 1986) : 433-450; B. Beatty, Preschool Education in America. The Culture of Young Children from the Colonial Era to the Present (New Haven : Yale University Press, 1995); Caroline Winterer, "Avoiding a "Hothouse System of Education": Nineteenth-Century Early Childhood Education from the Infant Schools to the Kindergartens", History of Education Quarterly 32, 3 (autumn 1992): 288-314; Kerstin Holmlund, "Child-cribs for the Poor and Kindergartens for the Rich: Two Directions for Early Childhood Institutions in Sweeden, 1854-1930", History of Education 28, 2 (1999): 143-155; Jean-Noël Luc, Linvention du jeune enfant au XIXe siècle. De la salle d'asile à l'école maternelle (Paris : Belin, 1997).

10 Allen, "Let Us Live with our Children", 25; Winterer, "Avoiding a 'Hothouse System of Education'”, 300.

11 Luc, Linvention du jeune enfant, 398-413; Winterer, "Avoiding a 'Hothouse System of Education."”

12 Allen, "Let Us Live with our Children", 29.

13 Dumont, "Des garderies ", 262-263, 273.

14 Archives des sœurs Grises de Montréal (ASGM), Fonds Asile Saint-Joseph.

Reproduction d'un article paru dans La Presse, 11 novembre 1899, cité dans Dumont, "Des garderies", 265.

15 ASGM, Journal de Sœur Julie Gaudry, p. 11-12, cité dans Dumont, " Des garderies », 266.

16 ASGM, Fonds asile Saint-Joseph. Reproduction d'un article paru dans La Presse, 11 novembre 1899, cité dans Dumont, " Des garderies ", 269-270.

17 Dumont, "Des garderies ", 271; Luc, L'invention du jeune enfant au XIXe siècle.

18 Sur les raisons qui expliquent la disparition des salles d'asile montréalaises voir Dumont "Des garderies", 281-284.

19 Archives de la Commission scolaire de Montréal ACSDM), autrefois Commission des Écoles catholiques de Montréal), Procès-verbaux des réunions des commissaires (PV), 13 et 27 décembre 1910, 26 décembre 1912, 9 janvier 1912, 26 mars 1912, 25 février 1913, 11 mars 1913, 13 et 27 mai 1913, 26 juin 1913 et 30 juillet 1913.

20 ACSDM, PV 23 avril 1912, 25 février 1913, 11 mars 1913, 13 et 27 mai 1913, 26 juin 1913 et 30 juillet 1913, 27 janvier 1914.

21 ACSDM, PV 16 septembre 1913 et 22 décembre 1914, 8 février 1915.

22 ACSDM, PV 10 novembre 1914, 22 décembre 1914 et 12 janvier et 8 février 1915.

23 De l'avis de l'inspecteur Miller qui visita l'École Gédéon-Ouimet en mai 1915, celle-ci ne pouvait d'ailleurs pas prétendre au titre d'école maternelle. Voir ACSDM, Monographie, École Gédéon-Ouimet (1942) et Rapport financier de la CÉCM pour l'exercice 1914-1915, 22.

24 ACSDM, PV 9 mars 1915. 
25 Ce programme se trouve à l'annexe $\mathrm{F}$ du règlement du comité catholique du Conseil de l'Instruction publique de la province de Québec refondu en 1915 (Morin, $\mathrm{La}$ maternelle, 7).

26 J.-C. Magnan, "Les écoles maternelles ", L'Enseignement primaire (EP) 37, 3 (nov. 1915), 129.

27 Ibid., 130.

28 Ibid.

29 Ibid., 132 et J.-C. Magnan, « Les écoles maternelles ", EP 37, 5 (janv. 1916), 261.

30 J.-C. Magnan, "Les écoles maternelles ", EP 37, 3 (nov. 1915), 129.

31 G. Daly, Les écoles maternelles, Brochure de l'École sociale populaire, 24 juin 1914, 4; « Des écoles maternelles », La Patrie, 29 mai 1926, 29.

32 Ibid., 5.

33 Ibid., 8.

34 Ibid., 9. Le souligné est de nous.

35 Ibid., 10.

36 Lettre du commissaire Eugène Lafontaine au curé Daly, 15 juin 1914, reproduite dans Les écoles maternelles, Brochure de l'ÉSP, 24 juin 1914, 25.

37 G. Daly, Les écoles maternelles, 7, 10; J.-C. Magnan, «Les écoles maternelles », EP 37, 5 (janv. 1916), 260.

38 Lettre du commissaire Eugène Lafontaine au curé Daly, 15 juin 1914, reproduite dans Les écoles maternelles, Brochure de l'ÉSP, 24 juin 1914, 22-23.

39 En décembre 1916, le commissaire Lafontaine parvient à faire adopter une résolution à l'effet que « la Commissions saisisse l'opportunité de la construction de nombreuses écoles sur son territoire pour établir des écoles maternelles " (ACSDM, PV 26 décembre 1916).

40 Les documents qui ont survécu ne permettent pas de dire ce qu'il est advenu de l'École Sainte-Anne, mais il est clair que l'École Gédéon-Ouimet a rapidement été transformée en école primaire. Selon l'abbé Philippe Perrier, des raisons financières expliqueraient que la CÉCM n'a pas été en mesure de développer son réseau de maternelles même si le nouveau programme des écoles publiques adopté en 1923 permettait leur création ( $\mathrm{La}$ Patrie, 29 mai 1926, 29, Gagnon, Histoire de la CÉCM, 113 et Louis-Philippe Audet, Histoire de l'enseignement au Québec (Montréal : Holt, Rinehart et Winston, 1971), t. 2., p. 273).

41 Cette association regroupait surtout des membres issus des classes moyennes. Elle s'était donné pour objectif de représenter tous les parents afin de mieux défendre leurs droits face aux clercs qui contrôlaient le système scolaire franco-catholique et prétendaient être les premiers éducateurs des enfants, mais elle cherchait aussi à accroître les compétences parentales, qu'elles jugeait déficientes, par la diffusion des nouvelles théories éducatives basées sur les préceptes de la psychologie moderne. Sur cette association, voir Denyse Baillargeon, "We Admire Modern Parents": The École des Parents du Québec and the Post-War Quebec Family, 1940-1959 ", dans Michael Gauvreau and Nancy Christie, ed., Cultures of Citizenship in Post-War Canada, 1940-1955 (Montréal \& Kingston : McGill-Queens University Press, 2003), 239-276 et Marie-Paule Malouin, Le mouvement familial au Québec. Les débuts : 1937-1965 (Montréal : Boréal, 1998), 31-36, 41-45 et 73-81.

42 Claudine Vallerand, "À l'intérieur d'une maternelle ", La Revue dominicaine, février 1939, 59.

43 Marie-Aimée Cliche, Maltraiter ou punir? La violence envers les enfants dans les familles québécoises 1850-1969 (Montréal : Boréal, 2007); Denyse Baillargeon, "We Admire Modern Parents"”.

44 Vallerand, "À l'intérieur d'une maternelle », 60.

45 Ibid., 71. 
46 Ibid.

47 Vallerand, "À l'intérieur d'une maternelle », 67.

48 Ibid., 71.

49 Vallerand, "Que penser de la maternelle? »; Jeannette Dalpé, « Pourquoi l'école avant 7 ans? ", L'École des Parents (ÉDP) 2, (février 1951) : 20-23; Christiane G. " Un mot sur les maternelles ", ÉDP 3, 2 (janv. 1952) : 10-13; Vallerand, "Un enfant comme bien d'autres ", ÉDP 6, 10 (oct. 1955): 17-18 et "L’enfant qui fréquente la maternelle ", ÉDP 7, 5 (mai 1956) : 15-16.

50 Le Devoir, 4 décembre 1940, p. 5.

51 Claudine-S. Vallerand, "École et Foyer», La Revue Dominicaine 45 (oct. 1939) : 142.

52 Ibid., 138.

53 Vallerand, "Que penser de la maternelle? ", Nos enfants, suppl. à la revue La Famille 1, 5 (avril 1941), 100 et "Avant d'apprendre à lire ", EDP 3, 2 (févr. 1952), 15.

54 ACSDM, Boîte 95, Étude et devoir à l'école, Lettre de parents au président de la CÉCM et aux commissaires, s. d. (1914).

55 "Les devoirs de classe à la maison », EP 45, 8 (avril 1924), 521-522; Abbé J. O. Maurice, "Le travail à domicile ", Causeries pédagogiques aux instituteurs de la CÉCM (Montréal : Arbour et Dupont, 1925), 107-120.

56 Maurice, "Le travail à domicile ", 117.

57 Ibid., 118-120.

58 Ibid., 18-120.

59 Emma-Maria Lalonde, «L'Étude à la maison », EP, 52 (7 mars 1931), 427.

60 Ibid., 428.

61 Ibid., 429.

62 Parents et maîtres. Leur collaboration, Brochure de l'École sociale populaire, 1928. Dans cette brochure l'auteur, demeuré anonyme, relie la question de la collaboration des parents avec l'école essentiellement à deux questions : les devoirs scolaires et la pratique religieuse.

63 ACSDM, Boîte 95, Étude et devoir à domicile, Lettre de J. H. Saucier à M.J.M. Manning, Directeur des Études, CÉCM, 4 décembre 1930.

64 ACSDM, Boîte 95, Étude et devoir à domicile, Lettre de $\mathrm{M}^{\mathrm{me}}$ Alyce Gagnon, novembre 1934.

65 ACSDM, Boîte 95, Étude et devoir à l'école, Lettre de M. et $\mathrm{M}^{\mathrm{me}}$ Lapierre à la CÉCM, 7 février 1935.

66 ACSDM, Boîte 95, Étude et devoir à la maison, Lettre de l'Association ouvrière du Nord à Victor Doré, président de la CÉCM, 6 février 1935; Étude et devoir à domicile, Lettre de l'échevin Rochon, quartier St-Michel, à Victor Doré, président de la CÉCM, 14 janvier 1934; Étude et devoir à domicile, 28 janvier 1935.

67 ACSDM, Boîte 95, Étude et devoir à domicile. Copie d'une résolution adoptée par la CÉCM, 23 novembre 1937.

68 ACSDM, Boîte 95, Étude et devoir à domicile. Lettre de Tancrède Marsil de l'agence générale de publicité, à Armand Dupuis, président de la CÉCM, 28 septembre 1937.

69 ACSDM, Boîte 95, Étude et devoir à domicile. Lettre de l'échevin Dave Rochon à Victor Doré, 16 février 1937; Lettre de J. E. Gariépy, secrétaire du CMTM à J. M. Manning, directeur des études, 9 octobre 1937.

70 ACSDM, Boîte 95, Étude et devoir à domicile, Service des études. Devoirs à domicile - Devoirs à l'école, chronologie.

71 "L'étude du soir abolie dans les écoles de Montréal ", L'Enseignement, septembre 1949, 5.

72 Mentionnons qu'un article percutant publié dans la revue Maclean's en 1951 et proposant l'abolition pure et simple des devoirs et des leçons, donne aussi à penser que la question était d'actualité un peu partout au Canada et même en Amérique du Nord. Sidney Katz, "Let's Abolish Homework », Maclean’s, $1^{\text {er }}$ octobre 1951. 
73 Rolande Major-Charbonneau, "Conditions dans lesquelles se fait l'étude à la maison», La Revue Pédagogie-Orientation 3, 5 (déc. 1949) : 347-351.

74 Ibid., 351.

75 ACSDM, Boîte 95, Étude et devoir à l'école. Comité catholique du Conseil de l'Instruction publique, Procès-verbal du 10 mai 1950. Soumission de rapports pour ou contre les devoirs à la maison.

76 Ibid.

77 Ibid.

78 «Voulez-vous bien vous fermer la boîte?», Le Front ouvrier, 13 octobre 1951, 6 et « Le foyer, milieu éducatif ", Le Front ouvrier, 20 octobre 1951, 2. Cette enquête révélait que $80 \%$ des enfants des familles ouvrières travaillaient dans la cuisine, au milieu du bruit des conversations ou au son de la radio.

79 «Voulez-vous bien vous fermer la boîte? ", Le Front ouvrier, 13 octobre 1951, 6.

80 "Le foyer, milieu éducatif ", Le Front ouvrier, 20 octobre 1951, 2.

81 "L'École et nous", ÉDP 2, 7 (juin 1951) : 15-18.

82 Vallerand, "Est-ce aussi votre avis? ", ÉDP 2, 10 (septembre 1951) : 22.

83 Ibid., 22.

84 Ibid., 23.

85 "Les devoirs à domicile : Pour ou contre? ", enquête de l'IP, L'enseignement 4, 9 (octobre 1950), 2; 4, 10 (novembre 1950), 8; 4, 12 (janvier 1951), 11; 5, 2 (mars 1951), 9; "Les devoirs à domicile ", L'enseignement 5, 2 (mai 1951), 9; "Supprimons les devoirs à domicile », 5, 10 (novembre 1951), 2.

86 "Les devoirs à domicile... Pour ou contre? ", L'enseignement 5, 11 (décembre 1951), 5.

87 Emma-Maria Lalonde, "Les devoirs à domicile ", L'enseignement 5, 11 (décembre 1951), 5. Le débat sur les devoirs a connu un certain regain à la fin des années 1950 pour emprunter à peu près les mêmes termes. Voir Guy Milot, conseiller d'orientation à la CÉCM, « Pour ou contre les devoirs à la maison »?, L'École canadienne 34, 6 (févr. 1959), 392-396 et Benoît Bussières, "Travail à domicile ", L'École canadienne 35, 4 (déc. 1959), XIII-XVI. 